\title{
Imaging spin flows in semiconductors subject to electric, magnetic, and strain fields
}

\author{
S. A. Crooker and D. L. Smith \\ Los Alamos National Laboratory, Los Alamos, NM 87545
}

(Dated: November 13, 2004)

\begin{abstract}
Using scanning Kerr microscopy, we directly acquire two-dimensional images of spin-polarized electrons flowing laterally in bulk epilayers of n:GaAs. Optical injection provides a local dc source of polarized electrons, whose subsequent drift and/or diffusion is controlled with electric, magnetic, and - in particular - strain fields. Spin precession induced by controlled uniaxial stress along the $\langle 110\rangle$ axes demonstrates the direct $\mathbf{k}$-linear spin-orbit coupling of electron spin to the shear (offdiagonal) components of the strain tensor, $\epsilon_{x y}$.

PACS numbers: 72.25.-b, 87.75.-d, 71.70.Ej, 72.25.Dc
\end{abstract}

The ability to control and measure electron spin degrees of freedom in semiconductors has been proposed as the operating principle for a new generation of novel electrical devices with the potential to overcome the power consumption and speed limitations of conventional electronic circuits 1, 2]. Semiconductor devices utilizing electron spin generally require: i) transport of spinpolarized electrons from one location in the device to another, and ii) a means to manipulate the electron spin orientation, either directly with magnetic fields or indirectly with electric and/or strain fields that exploit the spin-orbit interaction by coupling to electron orbital motion. Many spin-based semiconductor device proposals [3, 4, 5, 6] are based on a field-effect transistor geometry in which electron transport occurs in essentially 2dimensional structures. In order to design semiconductor structures whose function is based on electron spin it is necessary to understand the transport and flow of spinpolarized electrons, and how it is influenced by electric, magnetic and strain fields in these 2D structures.

Using methods for scanning Kerr microscopy, we acquire 2D images of spin-polarized conduction electrons flowing laterally in bulk epilayers of n-type GaAs. The images directly reveal the spatial dependence of spin diffusion and spin drift in the presence of applied electric, magnetic, and - in particular - strain fields. Controlled uniaxial stress along the $\langle 110\rangle$ axes induces spin precession, revealing the direct (k-linear) spin-orbit coupling of electron spin to the off-diagonal components of the strain tensor $\boldsymbol{\epsilon}$. The coupling may be characterized by an effective strain-induced magnetic field $\mathbf{B}_{\epsilon}$, which is shown to be orthogonal to the electron momentum $\mathbf{k}$, and therefore chiral for radially-diffusing spins. $\mathbf{B}_{\epsilon}$ scales linearly with $|\mathbf{k}|$, yielding a spatial precession of electron spins that is independent of electrical bias and is considerably more robust against the randomizing (ensemble dephasing) effects of spin diffusion as compared with precession induced by external magnetic fields.

The samples are $1 \mu \mathrm{m}$ thick, silicon-doped (n-type) GaAs epilayers grown by molecular beam epitaxy on [001]-oriented semi-insulating GaAs substrates. Doping densities are $\mathrm{n}_{e}=1,5$, and $10 \times 10^{16} / \mathrm{cm}^{3}$. Pieces were cleaved along the [110] and [110] natural cleave directions, and ohmic contacts allowed an in-plane, lateral electrical bias in the [110] direction. The samples were mounted in vacuum on the cold finger of an optical cryostat. To apply controlled uniaxial stress along the [110] or [11̄0] axis at low temperature, the cold finger incorporated a small cryogenic vise, whose lead screw was adjusted via a retractable actuator. Since all the samples exhibited qualitatively similar strain-related effects, we show data from only the $\mathrm{n}_{e}=10^{16} / \mathrm{cm}^{3}$ epilayer.

A local, steady-state source of electrons, spin polarized along the sample normal ([001], or $\hat{z})$, was provided by a circularly-polarized $1.58 \mathrm{eV}$ laser focused to a $4 \mu \mathrm{m}$ spot on the epilayer. While this pump laser (typically 10-25 $\mu \mathrm{W}$ ) injects $50 \%$ spin-polarized electrons and holes, the holes spin-relax and recombine rapidly, leaving behind a net spin polarization of the mobile conduction electrons [7]. These polarized electrons subsequently drift and/or diffuse laterally away from the point of generation. 2D images of the resulting $z$-component of electron spin polarization were acquired by measuring the polarization (Kerr) rotation imparted on a linearly-polarized probe laser that was reflected from the epilayer surface and raster-scanned in the $x-y$ epilayer plane. The probe beam $(50-100 \mu \mathrm{W})$ was derived from a narrowband tunable cw Ti:sapphire laser, and also focused to a $4 \mu \mathrm{m}$ spot. For lock-in measurement, the pump laser polarization was modulated from left- to right-circular (injecting spins along $\pm \hat{z})$ at $51 \mathrm{kHz}$.

The measured Kerr rotation is a strong function of probe laser energy near the GaAs bandgap (inset, Fig. 1a). Here, $30 \mu \mathrm{m}$ separates the pump and probe spots, so that the signal arises solely from a non-zero spin polarization of the electron Fermi sea. This energy-dependent response provides a relative (and in-situ) monitor of stressinduced bandedge shifts. When imaging, the probe energy is tuned (as shown) to the broad maximum that exists below the $1.515 \mathrm{eV}$ GaAs bandedge, to avoid perturbation of the Fermi sea.

Fig. 1a shows a $70 \times 140 \mu \mathrm{m}$ image of the steady-state electron spin polarization in the $\mathrm{n}_{e}=10^{16} / \mathrm{cm}^{3}$ GaAs epilayer due to spin diffusion alone. To emphasize smaller signals, the color scale has been adjusted so that white equals half the peak signal. The spatial extent of the measured spin polarization ( $\sim 60 \mu \mathrm{m}$ edge to edge $)$ is much larger than the focused pump laser (shown by the 


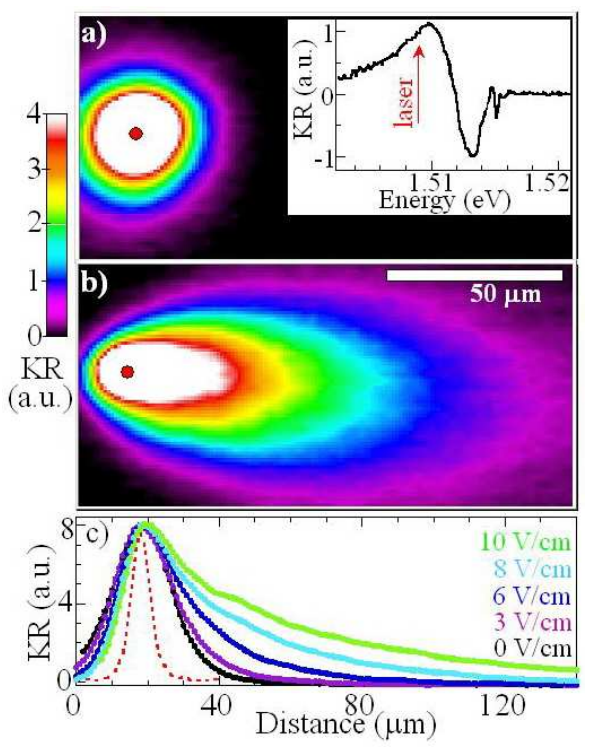

FIG. 1: (a) $70 \times 140 \mu \mathrm{m}$ image of electron spin polarization in a $1 \mu \mathrm{m}$ thick $\mathrm{n}$ :GaAs epilayer $\left(\mathrm{n}_{e}=10^{16} \mathrm{~cm}^{-3}\right)$ at $4 \mathrm{~K}$, acquired via Kerr-rotation (KR) microscopy. A circularly-polarized $1.58 \mathrm{eV}$ laser focused to a $4 \mu \mathrm{m}$ spot provides a local, $\mathrm{dc}$ source of spin polarized electrons; this image indicates $2 \mathrm{D}$ spin diffusion. Inset: KR vs. probe energy near $1.515 \mathrm{eV}$ GaAs bandedge. (b) With $\mathrm{E}=10 \mathrm{~V} / \mathrm{cm}$ lateral electrical bias, showing spin diffusion and drift. (c) Cross-sections of spin flow vs. bias; dotted line shows $5.5 \mu \mathrm{m}$ resolution.

red spot), indicating diffusion of electron spins away from the point of generation. Images of radially-diffusing electrons provide information on spin flows along all $\mathbf{k}$ directions in the $x-y$ sample plane, which will prove useful later in confirming the direction of $\mathbf{B}_{\epsilon}$. The characteristic diffusion length is given by the electron spin lifetime $\tau_{s}$ (measured independently via ultrafast techniques) and spin diffusion constant $D_{s}$. Fits to a $2 \mathrm{D}$ drift-diffusion model (described later) indicate $D_{s} \simeq 3$ and $15 \mathrm{~cm}^{2} / \mathrm{s}$ for the $\mathrm{n}_{e}=1$ and $5 \times 10^{16} / \mathrm{cm}^{3}$ samples at $4 \mathrm{~K}$ respectively, values in accord with the charge diffusion constants. Spin-polarized electrons can also be induced to drift laterally along an applied electric field E (Fig. 1b). This "spin drag", as revealed by Kikkawa and Awschalom [7], occurs over length scales $>100 \mu \mathrm{m}$. 2D images of spin drift and diffusion (Fig. 1b, where $\mathrm{E}=10 \mathrm{~V} / \mathrm{cm}$ ) show roughly elliptical contours of constant spin polarization, with major axis determined by electrical bias and lateral spin flow extending beyond $150 \mu \mathrm{m}$. Fig. 1c shows normalized line cuts through a series of images.

Spin-orbit coupling in GaAs permits coupling to electron spin degrees of freedom through the spatial part of the electron wavefunction, thus allowing induced precession of electron spins without external magnetic fields [8, 9, 10]. Spin-orbit effects lead to spin splittings of the conduction band along particular crystal momenta $\mathbf{k}$, and can be characterized by effective magnetic fields. Bulk inversion asymmetry (BIA) arises from the lack of



FIG. 2: (a-c) $80 \times 80 \mu \mathrm{m}$ images of $2 \mathrm{D}$ spin flow $(\mathrm{E}=10 \mathrm{~V} / \mathrm{cm})$ at $4 \mathrm{~K}$, showing induced spin precession with increasing [110] uniaxial stress. (d) KR vs. probe energy for the images, showing blueshift of GaAs bandedge with stress. (e) Line cuts through the images. Inset: Spatial frequency of spin precession vs. bandedge shift.

inversion symmetry in GaAs, leading to a spin-splitting for electrons with $\mathbf{k}$ along the $\langle 110\rangle$ axes (but no splitting along $\langle 111\rangle$ or $\langle 100\rangle$ axes). This ubiquitous coupling, cubic in $|\mathbf{k}|$, is the origin of the D'yakonov-Perel' mechanism of electron spin relaxation in bulk GaAs. A second spin-orbit effect arises from structural inversion asymmetry (the SIA or "Rashba" term [1]), as typically found in 2D heterostructures. Inversion asymmetry of the confining potential along the growth direction $\hat{z}$ (typically [001]) can often be characterized by an electric field $\mathbf{E}_{z}$, giving a Rashba Hamiltonian $H_{R} \propto \boldsymbol{\sigma} \cdot\left(\mathbf{k} \times \mathbf{E}_{z}\right)$. For typical $2 \mathrm{D}$ heterostructures with $\mathbf{k}$ in the $x-y$ plane, the effective magnetic field $\mathbf{k} \times \mathbf{E}_{z}$ is therefore in-plane and orthogonal to $\mathbf{k}$, with magnitude linear in $|\mathbf{k}|$. Control of the Rashba spin-orbit term, through a gate-tunable $\mathbf{E}_{z}$, is the basis of the original Datta-Das spin transistor [3].

Figure 2 demonstrates an additional spin-orbit effect; namely the coupling of the electron spin to the strain tensor $\boldsymbol{\epsilon}$. As described previously 12, 13, 14, 15, stress along the $\langle 110\rangle$ axes of GaAs induces k-linear spin splittings in the conduction band through the off-diagonal (shear) elements of $\boldsymbol{\epsilon}$. The strain Hamiltonian is $H_{S}=$ $c_{3} \sigma \cdot \varphi$, where $\left(\varphi_{x}, \varphi_{y}, \varphi_{z}\right)=\left(\epsilon_{x y} k_{y}-\epsilon_{x z} k_{z}, \epsilon_{y z} k_{z}-\right.$ $\left.\epsilon_{y x} k_{x}, \epsilon_{z x} k_{x}-\epsilon_{z y} k_{y}\right),(x, y, z)$ are the principle $\langle 100\rangle$ crystal axes, and the constant $c_{3}$ depends on the interband deformation potentials. Stress applied along the [110] or [11̄0] axis of GaAs gives in-plane shear $\epsilon_{x y}=\epsilon_{y x} \neq 0$. Thus for electrons moving in the $x-y$ plane, $H_{S}$ has similar symmetry to $H_{R}$, as it describes an in-plane effective magnetic field, orthogonal to $\mathbf{k}$, with magnitude linear in $|\mathbf{k}|$.

Figs. 2a-c show $80 \times 80 \mu \mathrm{m}$ images of steady-state electron spin flow (k $\|$ [110]) in the presence of increasing [110] uniaxial stress. Spin precession is observed, with 

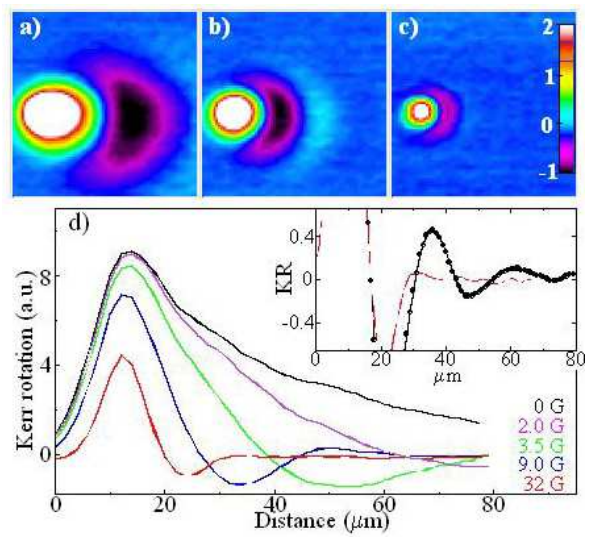

FIG. 3: (a-c) $80 \times 80 \mu \mathrm{m}$ images of $2 \mathrm{D}$ spin flow $(\mathrm{E}=10 \mathrm{~V} / \mathrm{cm})$ at $4 \mathrm{~K}$, with increasing applied magnetic field $\mathbf{B}_{a p p}=3.5,9$, and $32 \mathrm{G}$ along [110]. (d) Line-cuts through the images. Inset: Comparing line-cuts through Figs. 2c (black) and 3c (red).

increasing spatial frequency, indicating a strain-induced effective magnetic field $\mathbf{B}_{\epsilon} \propto \epsilon_{x y}|\mathbf{k}|$, oriented along [1110]. $\epsilon_{x y}$ is inferred from the measured blueshifts (see Fig. 2d) of $\sim 1$ and $2 \mathrm{meV}$ in Figs. $2 \mathrm{~b}$ and $2 \mathrm{c}$ respectively, indicating strain $\left|\epsilon_{x y}\right| \sim 1.5$ and $3.0 \times 10^{-4}$ (and applied stress $\sim 3.6$ and $7.2 \times 10^{8}$ dynes $\left./ \mathrm{cm}^{-2}[15]\right)$. These strains are small compared to typical $\sim 1 \%$ strains due to lattice mismatched growth; in fact, considerable care was required in sample mounting to avoid spurious and inhomogeneous strains during cool-down. With the cryogenic vise, the stress-induced precession of electron spins is controllable, reversible, and uniform over the sample. Line cuts along [110] (Fig. 2e) show many precession cycles ( $>5 \pi$ rotation). The inset of Fig. 2e confirms that the spatial frequency of the induced precession $\left(\propto \mathbf{B}_{\epsilon}\right)$ scales linearly with the observed bandshift $\left(\propto \epsilon_{x y}\right)$.

Only shear (off-diagonal) strain gives $\mathbf{k}$-linear spinorbit coupling to electron spins. Strain along the principle axes, either applied or arising from, e.g., lattice mismatched growth along [001], should not influence electron spins to leading order. However, shear strain and klinear coupling should exist in lattice-mismatched structures grown along [110] or [111]. Because shear strain reduces the symmetry of the zincblende GaAs crystal, these strain effects can be thought of as arising from BIA in a crystal of lower symmetry. In lower symmetry wurtzite crystals such as CdSe or $\mathrm{ZnO}$, BIA alone gives $\mathbf{k}$-linear spin splitting of electrons [16]. One should not think of $\mathbf{B}_{\epsilon}$ as arising from an electric field (e.g., a stress-induced piezoelectric field [17]), since any such field is screened in bulk metallic samples.

The line-cuts in Fig. 2e show that dc spin flows, precessing due to strain, are in phase at large distances from the point of generation. This robust behavior is in marked contrast with the rapid spatial dephasing that occurs when external magnetic fields are used to induce precession in dc spin flows, as shown in Fig. 3 Figs. 3 a-c show spin flows in the presence of an increasing ap-

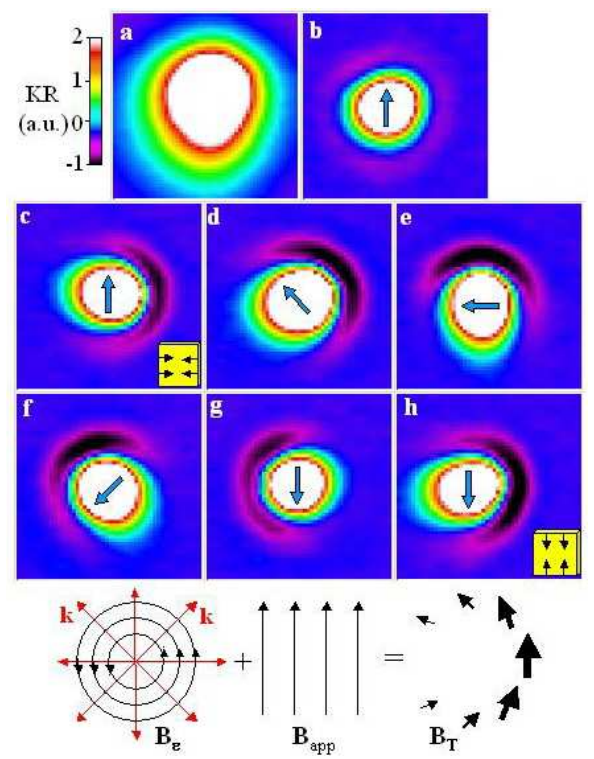

FIG. 4: $50 \times 50 \mu \mathrm{m}$ images of $2 \mathrm{D}$ spin diffusion $(\mathrm{E}=0)$ at $4 \mathrm{~K}$. (a) Stress $=0, \mathbf{B}_{a p p}=0$. (b) Stress $=0, \mathbf{B}_{a p p}=16 \mathrm{G}$ oriented along [1ํㅣ as shown. (c) $\mathbf{B}_{a p p}=16 \mathrm{G}$, with [110] uniaxial stress. Spins diffusing to the right precess, while those diffusing to the left do not. Thus $\mathbf{B}_{\epsilon}$ is chiral for radially-diffusing electrons (see diagram). (d-g) Maintaining [110] stress, $\mathbf{B}_{a p p}$ is rotated by 180 degrees in-plane. (h) Stress is switched to [110], reversing chirality of $\mathbf{B}_{\epsilon}$.

plied magnetic field $\left(\mathbf{B}_{a p p} \|[1 \overline{1} 0]\right)$, with line-cuts in Fig. $3 \mathrm{~d}$. As $\mathbf{B}_{a p p}$ increases, the ensemble spin polarization becomes dephased at distances beyond one precession period, particularly when the precession period falls below the spin diffusion length. This pronounced spatial dephasing of dc spin flows is due to the randomizing nature of diffusion. The net spin at a remote location is the combined sum of many random walks. Each path takes a different amount of time, giving a different degree of spin precession, leading to dephasing. Future spintronic devices based on magnetic field manipulation of diffusive spin flow may thus be practically limited to a regime requiring $\pi$ rotation or less.

A comparison of linecuts through Figs. 2c and 3c are shown in the inset of Fig. 3d. Clearly, the spatial coherence of dc spin flows is preserved over more precession cycles (and greater distance) when the spins are manipulated with strain instead of magnetic field. This is a direct consequence of the $|\mathbf{k}|$-linear nature of $\mathbf{B}_{\epsilon}$, which correlates precession frequency with electron velocity (and therefore position). Indeed, if electrons moved along only one dimension, the spin flow would not dephase at all (it would still, of course, decohere). Scattering from $+\mathbf{k}$ to -k would simply reverse the direction of precession, leading to an exact correspondence between spatial position and spin orientation.

The images in Fig. 4 confirm that $\mathbf{B}_{\epsilon}$ is orthogonal to $\mathbf{k}$, such that $\mathbf{B}_{\epsilon}$ circulates around the point of injection for radially diffusing electrons. Image (a) shows 


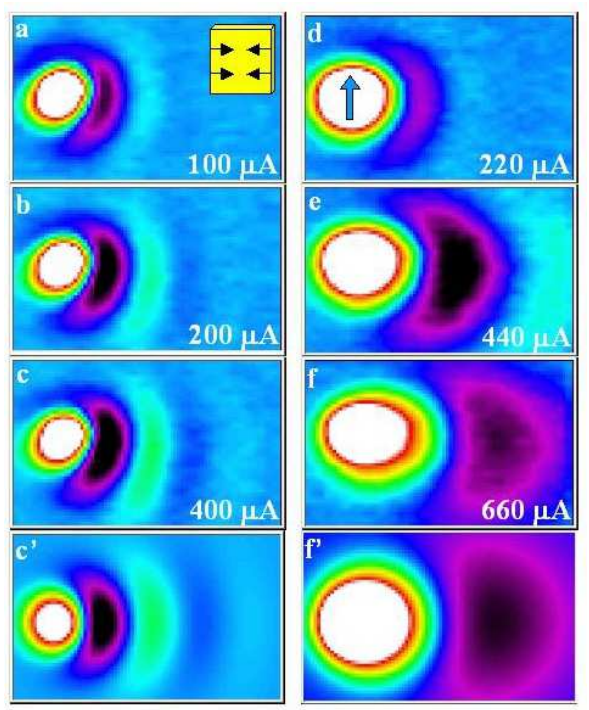

FIG. 5: $50 \times 80 \mu \mathrm{m}$ images of $2 \mathrm{D}$ spin flow at $4 \mathrm{~K}$ with increasing $|\mathbf{k}|$ (current). (a-c) With [110] stress and $\mathbf{B}_{a p p}=0$. Spatial period of precession is fixed. (d-f) Stress $=0, \mathbf{B}_{a p p}=6$ G; spatial period varies. Images c') and f') are simulations of c) and f), using the model described in text.

unperturbed spin diffusion (no stress and $\mathbf{B}_{a p p}=0$ ). In (b), $\mathbf{B}_{\text {app }}=16 \mathrm{G}$ along [110] as indicated. Spins precess uniformly, regardless of $\mathbf{k}$, giving a faint annulus of oppositely oriented spins (negative signal) surrounding the injection point. In (c), stress is applied along [110], and the image becomes asymmetric. Electrons diffusing to the right along $\mathbf{k} \|[110]$ undergo precession, while those diffusing to the left along $-\mathbf{k}$ do not. That is, the total field $\left(\mathbf{B}_{T}=\mathbf{B}_{\epsilon}+\mathbf{B}_{a p p}\right)$ is finite for spins diffusing to the right, but $\mathbf{B}_{T}$ is effectively zero for spins diffusing to the left. This image is consistent with a uniform $\mathbf{B}_{a p p}$ added to a circulating $\mathbf{B}_{\epsilon}$, as shown. Maintaining [110] stress, the image asymmetry rotates and ultimately reverses as $\mathbf{B}_{a p p}$ is rotated in the $x-y$ plane through 180 degrees (c-g). Finally (h), when stress is applied along the [110] axis, the asymmetry again reverses, indicating the opposite chirality of $\mathbf{B}_{\epsilon}$.
For comparison with data, we derive and solve the spin drift-diffusion equations that describe dc spin flows in the presence of electric, magnetic and strain fields. For simplicity, the [110] strain axis is taken here to be the $x$-axis, and the electric and magnetic fields are in the $x$ - $y$ sample plane. The spin polarization is described by the $\left(\rho_{x}, \rho_{y}\right.$, $\rho_{z}$ ) components of a $2 \times 2$ density matrix, where $\rho_{z}$ gives the ensemble spin density. The equations are $O_{1} \rho_{x}=$ $-O_{2} \rho_{z}, O_{1} \rho_{y}=-O_{3} \rho_{z}$, and $O_{4} \rho_{z}-O_{2} \rho_{x}-O_{3} \rho_{y}=-G_{z}$, with operators $O_{1}=D \nabla^{2}+\mu \mathbf{E} \cdot \nabla-\left(C_{s} \varepsilon\right)^{2} D-1 / T_{2}, O_{2}=$ $-C_{B} B_{y}+C_{s} \varepsilon\left(2 D \nabla_{x}+\mu E_{x}\right), O_{3}=C_{B} B_{x}+C_{s} \varepsilon\left(2 D \nabla_{y}+\right.$ $\left.\mu E_{y}\right)$, and $O_{4}=D \nabla^{2}+\mu \mathbf{E} \cdot \nabla-2\left(C_{S} \varepsilon\right)^{2} D-1 / T_{1} . D$ is the spin diffusion constant, $\mu$ is the electron mobility, $T_{2}$ and $T_{1}$ are transverse and longitudinal spin lifetimes, the magnetic coupling constant is $C_{B}=\frac{g \mu_{B}}{\hbar}$, the strain coupling constant $C_{s}$ is given in Ref.[15], $G_{z}$ is the spin generation rate, and $\varepsilon$ is the off-diagonal strain element $\epsilon_{x y}$. Drift and diffusion appear in the strain coupling terms, due to its dependence on $\mathbf{k}$. The $1 \mu \mathrm{m}$ thick $\mathrm{n}: G a A s$ layer is small compared with a lateral spin diffusion length, thus, the problem is 2-dimensional. The equations are solved with numerical Fourier transform methods.

In contrast with the case of an applied magnetic field, both the model and the data reveal a consequence of $\mathbf{k}$ linear $\mathbf{B}_{\epsilon}$ : the spatial period of precession is independent of applied electrical bias. Fig. 5 confirms this, showing spin flow along [110]. In the presence of strain (Fig. $5 \mathrm{a}-\mathrm{c}$ ), the spatial precession period is independent of electrical bias, whereas for the case of applied magnetic field (d-f), the spatial precession period clearly increases with increasing electrical bias. Functional devices based on rotation of spin from one point in space to another (e.g., from source to drain contacts in spin transistor designs $[3,4,[5,6]$ ) may well benefit from the freedom to operate at variable electrical bias. Along with spin manipulation via Rashba coupling (also linear in $|\mathbf{k}|)$, the spin-orbit coupling of spin flows to shear strain, as detailed in this work, also affords this flexibility.

This work was supported by the DARPA SpinS and the Los Alamos LDRD programs. We thank S. Kos, P. Littlewood, and I. Martin for valuable discussions.
[1] S. A. Wolf et al., Science 294, 1488 (2001).

[2] I. Žutić, J. Fabian, and S. Das Sarma, Rev. Mod. Phys. 76, 323 (2004).

[3] S. Datta and B. Das, Appl. Phys. Lett. 56, 665 (1990).

[4] J. Schliemann, J. C. Egues, and D. Loss, Phys. Rev. Lett. 90, 146801 (2003).

[5] K. C. Hall et al., Appl. Phys. Lett. 83, 2937 (2003).

[6] X. Cartoixà, D. Z.-Y. Ting, and Y.-C. Chang, Appl. Phys. Lett. 83, 1462 (2003).

[7] J. M. Kikkawa and D. D. Awschalom, Nature 397, 139 (1999).

[8] Y. Kato, R. C. Myers, A. C. Gossard, and D. D. Awschalom, Nature 427, 50 (2004); ibid, Phys. Rev. Lett.
93, 176601 (2004).

[9] M. E. Flatté, J. M. Byers, and W. H. Lau, in Semiconductor Spintronics and Quantum Computation, edited by D. D. Awschalom, D. Loss, and N. Samarth (Springer, Berlin, 2002).

[10] R. Winkler, Spin-Orbit Coupling Effects in 2D Electron and Hole Systems (Springer, Berlin, 2003).

[11] Y. A. Bychkov and E. I. Rashba, J. Phys. C 17, 6039 (1984).

[12] G.E. Pikus and A.N. Titkov, in Optical Orientation, edited by F. Meier and B. P. Zakharchenya (NorthHolland, Amsterdam, 1984).

[13] D. G. Seiler, B. D. Bajaj, and A. E. Stephens, Phys. Rev. 
B 16, 2822 (1977).

[14] M. Cardona, V. A. Maruschak, and A. N. Titkov, Solid State Comm. 50, 701 (1984); M. Cardona, N. E. Christensen, and G. Fasol, Phys. Rev. B 38, 1806 (1988).

[15] G. L. Bir and G.E. Pikus, Symmetry and Strain-Induced
Effects in Semiconductors (Halstead, New York, 1974).

[16] L. C. L. Y. Voon, M. Willatzen, M. Cardona, and N. E. Christensen, Phys. Rev. B 53, 10703 (1996).

[17] K. Fricke, J. Appl. Phys. 70, 914 (1991). 